\section{Thyroid storm presenting as status epilepticus and stroke}

Sir,

Patients with thyroid storm typically present with hyperthermia, tachycardia, and central nervous system dysfunction such as confusion, lethargy, and psychosis culminating in stupor and coma. Very rarely, repeated stroke is the sole clinical manifestation. ${ }^{1}$ To our knowledge, there has been only report of status epilepticus during the clinical course of thyroid storm. ${ }^{2}$ We experienced a woman with thyroid storm who initially presented with status epilepticus followed by stroke.

A 56-year-old woman was taken to the emergency room as a result of recurrent generalised seizures which had occurred four times for an hour on the day of admission. After the first seizure at home, she had become confused and lethargic. On arrival at hospital, she was still confused and irritable, and then she experienced her fifth generalised seizure of the day. After this seizure, intravenous sodium valproate $(1200 \mathrm{mg} /$ day $)$ was started. The history revealed Graves' disease, diagnosed three years earlier, although she had discontinued antithyroid medication about two years before. In the past year, she had had a brief episode of frank psychosis. For the most recent three days, she had reported upper respiratory symptoms. Examination revealed tachycardia (140 beats $/ \mathrm{min}$ ), sweating, and an enlarged thyroid. Neurological evaluation showed confusion, irritability, generalised rigidity, and neck stiffness. Enhanced computed tomography (CT) and cerebrospinal fluid study were normal. Electrocardiographic monitoring documented persistent sinus tachycardia.

Immediately after the brain CT scan, she was found to have right hemiplegia and she became stuporous. The attending physician then managed her as a thyroid storm. Thyroid function test showed free thyroxine $59.6 \mathrm{pmol} / 1$ (normal 9-24) and thyroid-stimulating hormone $0.05 \mathrm{mU} / 1$ (normal 0.4 to 0.6). An electroencephalogram (EEG) showed diffuse slow waves in the delta and theta range. In the next morning, she developed a high fever that lasted for three days. A $\mathrm{T}_{2}$-weighted magnetic reasonance imaging (MRI) of the brain showed a small lacune in the left fronal subcortical white matter. MR angiography (MRA) showed mild stenosis at the bilateral terminal internal carotid arteries and the left middle cerebral artery.

Ticlopidine was started to prevent ischaemic stroke. On the fourth day, single-photon-

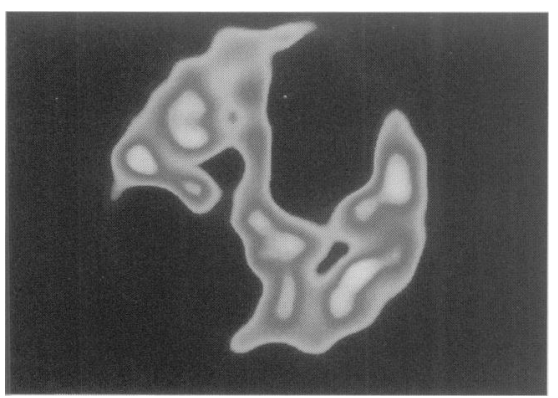

Figure 1 emission computed tomography (SPECT) using 99m-Tc-HMPAO showed perfusion defects in the right fronto-parietal and left frontal areas of the brain. The follow-up MRI on the tenth day revealed cerebral infarcts including watershed areas between the right middle and posterior cerebral artery territories, and between the left anterior and middle cerebral artery territories. The follow-up SPECT and MRA showed no changes. However, serial thyroid function tests and the follow-up EEG showed marked improvement. An extensive evaluation for stroke risk factors showed no abnormality. In the fifth week, she was discharged home in satisfactory condition.

Having uncontrolled Graves' disease and a superimposed respiratory infection, our patient with thyroid storm initially presented as status epilepticus. Both status epilepticus and stroke are very rare in thyroid storm. ${ }^{1,2}$ Possibly, the past history of brief psychosis might also be thyroid storm. We could not completely exclude the possibility that status epilepticus caused thyroid storm in our patient, although theorectically this is almost nil.

Since $1956,{ }^{3}$ seizures secondary to thyrotoxicosis have rarely been reported. Experimentally, in mice, thyroxine has been shown to lower the seizure threshold. ${ }^{4}$ In the previous report of thyroid storm with status epilepticus, the seizures were not controlled by diazepam, but responded to chlormethimazole. ${ }^{2}$ In contrast, the seizures in our patient were well controlled with sodum valproate and did not recur thereafter.

The hemiplegia after enhanced CT scan cannot be sufficiently explained by the initial MRI finding of the lacune. Subsequently, brain SPECT revealed large perfusion defects which were not compatible with the major arterial territories. We suppose that these were related to the metabolic derangement (increased metabolic demand) and aggravated by iodinated contrast dye which precipitates thyroid storm. In a report of thyroid storm with recurrent stroke, despite the lack of the embolic source, the authors

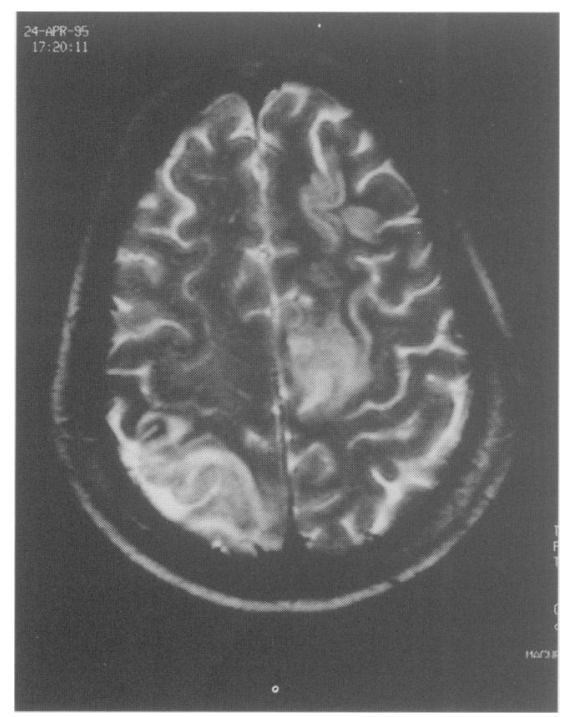

Figure 2 suggested thromboembolic events recurring after stopping heparin. ${ }^{1}$ In contrast, our patient recovered without heparin, and arterial vasospasm could be excluded by MRA. However, venous infarction or vasculitis could not be excluded. Hitherto the exact mechanism(s) of ischaemic stroke in thyroid storm remains uncertain.

TG LEE

The Headache Center, The Cleveland Clinic Foundation, 9500 Euclid Avenue, Cleveland, OH 44195, USA CK HA BH LIM

Department of Neurology, Gyeong-Sang National University Hospital, finju City,

Korea

1 Jarrett DRJ, Hansell DM, Zeegen R. Thyroid crisis complicated by cerebral infarction. $\mathrm{Br} \mathcal{F}$ Clin Pract 1987; 41: 671 -3.

2 Safe AF, Griffith KD, Maxwell RT. Thyrotoxic crisis presenting as status epilepticus. Postgrad Med f 1990; 66: 150-2.

3 Skanse B, Nyman G. Thyrotoxicosis as a cause of cerebral dysrhythmia and convulsive seizures. Acta Endocrinol (Copenh) 1956; 22: 246-63.

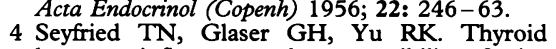
4 Seyrried TN, Glaser GH, Yu RK. Thyroid hormone influence on the susceptibility of mice 600 .

\section{Endocarditis caused by Lactobacillus}

Sir,

In the absence of intravenous substance use, $\alpha$-haemolytic streptococci remain the most common aetiology of infective endocarditis. A variety of uncommon organisms may also cause endocarditis, however. We report a case of endocarditis due to Lactobacillus. Only a few cases of endocarditis related to this organism have been reported..$^{1-3}$

An 80-year-old woman with a past history of hypertension and dementia was admitted to our Medical Center with shortness of breath of unknown duration. Physical examination revealed an afebrile patient with poorly maintained dentition, a Gr III/VI pansystolic murmur at the apex radiating to the axilla, and bibasilar rales. No peripheral signs of infective endocarditis were present. The chest $\mathrm{X}$-ray revealed mild pulmonary oedema and cardiomegaly. An electrocardiogram showed atrial fibrillation.

On the second hospital day, the patient was noted to have a temperature of $101^{\circ} \mathrm{F}$ with no change in physical findings. A blood culture drawn on that day and one on the following day were found to be positive for Lactobacillus jensenii. A transoesophageal echocardiogram revealed a large vegetation on the aortic valve with moderate to severe mitral regurgitation and mild to moderate aortic regurgitation. The patient was given ampicillin $(2 \mathrm{~g}$ intravenously every four hours) and gentamicin (60 mg every eight hours ( $3 \mathrm{mg} / \mathrm{kg}$ daily) for four weeks. Follow-up blood cultures drawn during and after treatment remained sterile.

Lactobacillus species are ubiquitous and generally considered non-pathogenic inhabitants of the oral cavity, gastrointestinal tract and female genital tract. Review of 23 reported cases of Lactobacillus endocarditis shows that it typically occurs in patients with pre-existing heart disease and often with some 